\title{
Negative cooperativity in NAD(P)H quinone oxidoreductase 1 (NQO1)
}

\author{
Clare F. Megarity ${ }^{[a]}$, Hoda Abdel-Aal Bettley ${ }^{[b]}$, M. Clare Caraher ${ }^{[a],[b]}$, Katherine A. Scott ${ }^{[b]}$, Roger C. \\ Whitehead ${ }^{[c]}$, Thomas A. Jowitt ${ }^{[d]}$, Aldo Gutierrez ${ }^{[e]}$, Richard A. Bryce ${ }^{[b]}$, Karen A. Nolan ${ }^{[b]}$, Ian J. \\ Stratford $^{[\mathrm{b}]}$, David J. Timson ${ }^{*[\mathrm{a}],[\mathrm{f}]}$
}

\begin{abstract}
NAD}(\mathrm{P}) \mathrm{H}$ quinone oxidoreductase-1 (NQO1) is a homodimeric protein that acts as a detoxifying enzyme or as a chaperone/nanny protein. Dicourmarol interacts with NQO1 at the $\mathrm{NAD}(\mathrm{P}) \mathrm{H}$ binding site and can both inhibit enzyme activity and modulate the interaction of NQO1 with other proteins. We show the binding of dicoumarol and related compounds to NQO1 generates negative cooperativity between the monomers. This does not occur in the presence of the reducing co-factor, $\mathrm{NAD}(\mathrm{P}) \mathrm{H}$, alone. Alteration of Gly150 (but not Gly149 or Gly174) abolished the dicoumarol-induced negative cooperativity. Analysis of the dynamics of NQO1 with the Gaussian network model indicates a high degree of collective motion by monomers and domains within NQO1. Ligand binding is predicted to alter NQO1 dynamics both proximal to the ligand binding site and remotely, close to the second binding site. Thus, drug-induced modulation of protein motion may contribute to the biological effects of putative inhibitors of NQO1.
\end{abstract}

\section{Introduction}

$\mathrm{NAD}(\mathrm{P}) \mathrm{H}$ quinone oxidoreductase (NQO1, DT-diaphorase, E.C. 1.6.5.2) is a homodimeric flavoprotein with one molecule of non-covalently bound flavin adenine dinucleotide (FAD) per monomer. ${ }^{[1]}$ The enzyme's two active sites are located at the interface between the two subunits and involve residues from both polypeptide chains. ${ }^{[1 \mathrm{a}]} \mathrm{NQO} 1$ is a detoxification enzyme with its main function being the obligate two-electron reduction of quinones to hydroquinones. ${ }^{[2]}$ The enzyme is unusual in that it can use either NADH or NADPH in the first step of its catalytic process the transfer of a hydride group to the FAD molecule. $\left.{ }^{[1 a}, 3\right]$ The second step is the transfer of the hydride group from the reduced cofactor to the newly docked quinone substrate, but this can only occur once the $\mathrm{NAD}(\mathrm{P})^{+}$has been released, hence the description of the mechanism as a "ping-pong" reaction. ${ }^{[1 a, 3-4]}$ It has been

[a] Dr Clare F. Megarity, Dr Mary Clare Caraher, Dr David J. Timson, School of Biological Sciences, Queen's University Belfast, Medical Biology Centre, 97 Lisburn Road, Belfast, BT9 7BL, UK

[b] Dr Hoda Abdel-Aal Bettley, Dr M. Clare Caraher, Dr Katherine Scott, Dr Richard A. Bryce, Dr Karen A. Nolan, Prof lan J. Stratford, Manchester Pharmacy School, [c] Dr Roger C. Whitehead, School of Chemistry, and [d] DrThomas A. Jowitt, The Faculty of Life Science, Manchester Cancer Research Centre and the University of Manchester, Oxford Road, Manchester, M13 9PT, UK

[e] Dr Aldo Gutierrez, Department of Biosciences, Nottingham Trent University, Nottingham NG1 4BU, UK

[f] Prof David J Timson, School of Pharmacy and Biomolecular Sciences, University of Brighton, Huxley Building, Lewes Road, Brighton, BN2 4GJ, UK; +44(0)1273 641623;

d.timson@brighton.ac.uk

Supporting information for this article is given via a link at the end of the document. suggested that the two active sites in the enzyme act independently ${ }^{[1 \mathrm{a}]}$. However, in an early study of NQO1 from rat liver, enzyme kinetic analysis indicated non-equivalent active sites and negative cooperativity towards some inhibitors. ${ }^{[5]}$ Recent work combining biophysical and computational methods has demonstrated considerable effects of mobility within the enzyme. Although the C-terminal domain (CTD) is not directly involved in forming the active site, removal of this part of the protein dramatically reduces the affinity for FAD and, consequently, catalytic activity. ${ }^{[6]}$ The binding of FAD to one active site reduces the affinity at the other. ${ }^{[7]}$ This negative cooperativity of cofactor binding may have implications for catalysis and the binding of other ligands ${ }^{[8]}$.

NQO1 has also been shown to have chaperone properties. It stabilises the tumour suppressor protein p53 and other short-lived proteins such as $p 73 \alpha$ and ornithine decarboxylase..$^{[9]}$ This stabilization phenomenon is $\mathrm{NAD}(\mathrm{P}) \mathrm{H}$-dependent and inhibitors of NQO1, such as dicoumarol and curcumin, which compete with $\mathrm{NAD}(\mathrm{P}) \mathrm{H}$, have been shown to promote $\mathrm{p} 53$ degradation. ${ }^{[9 c, 10]}$

The enzyme is overexpressed in some cancer cells and inhibition of NQO1 has been proposed as a cancer chemotherapy strategy. The increased NQO1 activity in these cells may be associated with enhanced drug detoxification, increased stress resistance and decreased susceptibility to p53-mediated apoptosis. ${ }^{[11]}$ Dicoumarol has been shown to inhibit the growth of pancreatic cancer cells. ${ }^{[12]}$ However, this compound is not an ideal anticancer agent due to its off target effects which include anticoagulant and mitochondrial "uncoupling" activity.[13] Consequently a number of studies have focussed on identifying novel inhibitors of NQO1, often ones which are structurally similar to dicoumarol 1 (Scheme 1) or quinones, for example see reference ${ }^{[14]}$. Conversely, reduced NQO1 activity resulting from polymorphic forms of NQO1, the most common of which results in a proline to serine substitution at position 187, can result in genetic predisposition to certain types of cancer, presumably due to reduced cellular antioxidant activity. ${ }^{[15]}$ This $\mathrm{P} 187 \mathrm{~S}$ variant has reduced affinity for FAD and increased susceptibility to proteolytic degradation. ${ }^{[6 a, 6 b, 16]}$

The importance of NQO1 in detoxification, molecular pathology and as a potential drug target all mean that understanding the enzyme's interaction with ligands is critical. The potential for cooperativity in the enzyme suggests communication between the active sites. These phenomena are likely to have effects beyond enzymology and may impact on NQO1's cellular roles as a chaperone. Here, we demonstrate kinetic negative cooperativity of human NQO1 by dicoumarol and three structurally related inhibitors (compounds 1-4; Scheme 1). Overall, our results paint a picture of an enzyme in which modulation of protein motions mediates communication between the two active sites.

For internal use, please do not delete. Submitted_Manuscript 
<smiles>CC(C)Cc1c(O)c2ccccc2oc1=O</smiles><smiles>Cc1cc2oc(=O)c(Cc3ccc4ccccc4c3)c(O)c2cc1C</smiles><smiles></smiles>

$3\left(\mathrm{I}_{\mathrm{sc}}=2.2 \mathrm{nM}\right)$

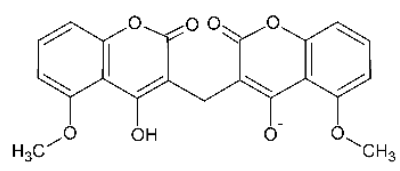

$4\left(\mathrm{IC}_{50}=2.8 \mathrm{nM}\right)$

Scheme 1: The compounds used in this study. All four of these compounds have been shown to inhibit NQO1 in vitro and the growth of HT29 cells in culture. IC $C_{50}$ values have been reported previously. ${ }^{14}$ Compound 1 is dicoumarol. Compounds 2, 3 and 4 were designated AS1, AS3 and S4 in previous work. ${ }^{[14 a, 14 c]}$

\section{Results and Discussion Cellular inhibition of NQO1 results in lower p53 levels}

Previous studies have shown that dicoumarol (1) inhibits NQO1 in vivo and, consequently, reduces the amount of the apoptosis regulator $p 53 .{ }^{10 a, 14 c, 17]}$ Three new analogues of 1 have been described (2-4; Scheme 1) which also inhibit NQO1 in vitro. ${ }^{[14 a]}$ These inhibitors also reduce the amount of cellular p53 in the presence and absence of etoposide (a p53 inducer) at concentrations similar to those required to achieve enzyme inhibition (Figure S1, S2, S3). The concentrations of 2-4 were below those which cause toxicity in the cell lines used. ${ }^{[14 a, 14 c]}$ Thus these newly identified inhibitors have promise for the pharmacological inhibition of NQO1 and associated reduction in p53 levels with reduced side-effects compared to 1.

\section{Human NQO1 shows negative cooperativity towards inhibitors, but not towards $N A D(P) H$}

In the 1970s, it was reported that rat NQO1 shows negative cooperativity towards some inhibitors, including $1 .^{[5]}$ More recently negative cooperativity towards inhibitors has been observed in the functionally related proteins human $\mathrm{NRH}$ quinone oxidoreductase 2 (NQO2; EC 1.10.5.1) and Saccharomyces cerevisiae Lot6p (EC 1.5.1.39). ${ }^{[18]}$ Here we find that recombinant human NQO1 showed negative cooperativity towards 1 and also towards 2-4: Hill plots of inhibition data revealed Hill coefficients of approximately 0.5 and $\Delta \Delta \mathrm{G}$ values between 4.9 and $7.3 \mathrm{kJmol}^{-}$ 1 for all four compounds (Figure 1; Table 1). Interestingly no cooperativity was observed towards the substrates NADH and NADPH, despite 1-4 competitively inhibiting NQO1 with respect to these compounds ${ }^{[14 a, 19]}$ (Table 1; Figure S4). Negative cooperativity towards 1-4 was confirmed by direct binding experiments using isothermal titration calorimetry. The binding of ligands to the dimeric NQO1 was best fitted to a "sequential binding sites" model (Figure S5).

\section{Negative cooperativity in NQO1 can be disrupted by a single amino acid substitution}

Negative cooperativity implies that there is some form of communication between the active sites. This can be mediated by conformational changes or through alterations in the mobility of elements of the protein's structure. ${ }^{[20]}$ Since glycine residues permit the greatest flexibility of a polypeptide chain, we concentrated on these residues as possible places to disrupt communication between the active sites. ${ }^{[21]}$ We focussed on three residues, Gly149, Gly150 and Gly174. These amino acids are in proximity to the dicoumarol binding site and Gly174 breaks a-helix a4 (following the notation of Faig et al.) ${ }^{[14 \mathrm{~g}]}$ which spans the distance between the active sites (residues 163-180). ${ }^{[22]}$

Alteration of Gly149 to serine resulted in no change in the Hill coefficient for ligand 1. When Gly174 was changed to serine, the purified protein was noticeably less yellow than the wild-type (suggesting a failure to bind FAD) and no activity could be detected. Supplementation of the reaction with FAD restored activity, but the Hill coefficient was similar to that of wild-type. However, altering Gly150 to serine increased $h$ to almost one in both the presence and absence of exogenous FAD $(\Delta \Delta G$ approximately $1 \mathrm{kJmol}^{-1}$ ) (Figure $1 \mathrm{~b}$ and Table 1 ). Like the wildtype protein, all three glycine-to-serine substitutions showed no cooperativity towards NADH (Figure S4 and Table 1). The serinesubstituted variants showed similar stability towards limited proteolysis (Figure S6) and only slightly increased susceptibility to thermal denaturation (as judged by differential scanning fluorimetry; Table 1).

\section{Elastic network modelling of NQO1 supports a dynamical basis for negative cooperativity}

In order to explore the structural basis for these observations, with a particular focus on the modulation of cooperativity within NQO1, we described the enzyme using a coarse grained dynamical approach, namely the Gaussian network model (GNM). ${ }^{[23]}$ Such elastic network models have been widely used in characterising the global dynamics and allostery of proteins and their complexes. ${ }^{[24]}$ In a GNM of a protein, the residues, along with cofactor and ligand where applicable, are connected by harmonic springs. The low frequency modes of motion derived from the resulting connectivity matrix can indicate key mechanical and functional features of a protein.

Here we consider GNM analysis based on the X-ray structure of NQO1 in complex with ligand 1, with corresponding PDB code $2 \mathrm{~F} 1 \mathrm{O} .{ }^{[22]}$ Each monomer of NQO1 comprises a large N-terminal domain (NTD) with flavodoxin topology, involving residues 1-223 and a smaller CTD, from residues 224-273; FAD and ligand bind at the interface between the monomers (Figure 2a). Overall, the NQO1 dimer becomes slightly more compact on binding 1-4, as demonstrated by analytical ultracentrifugation (Figure S7, Table S1). For NQO1, computation of B-factors via the Gaussian network model indicates areas of mobility; these indicate higher flexibility around residues $240-248$ in each monomer. This region corresponds to the two short helices, $\alpha 8$, of the CTD (Figure 2a,b). Other regions of high $\mathrm{B}$-factor value involve amino acids at the $\alpha 1-\beta 2$ junction $(31-35)$, loop L3 (58 - 68), loop L9 (230 - 235), as well as CTD helix $\alpha 5(197-211)$. These predicted high Bfactor regions correspond well to those found experimentally (Figure 2a,b). 
(a)
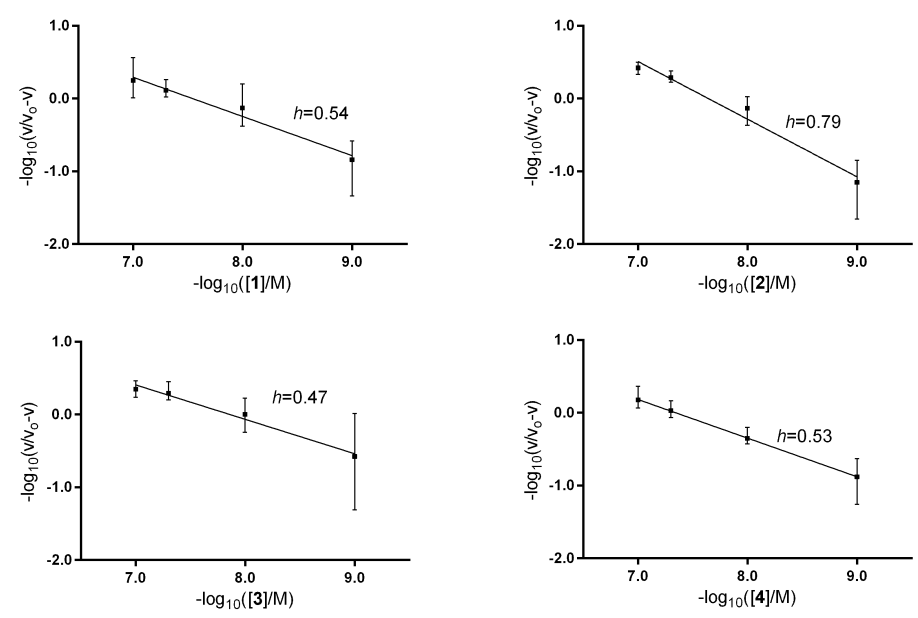

(b)

$-F A D$

$+\mathrm{FAD}$
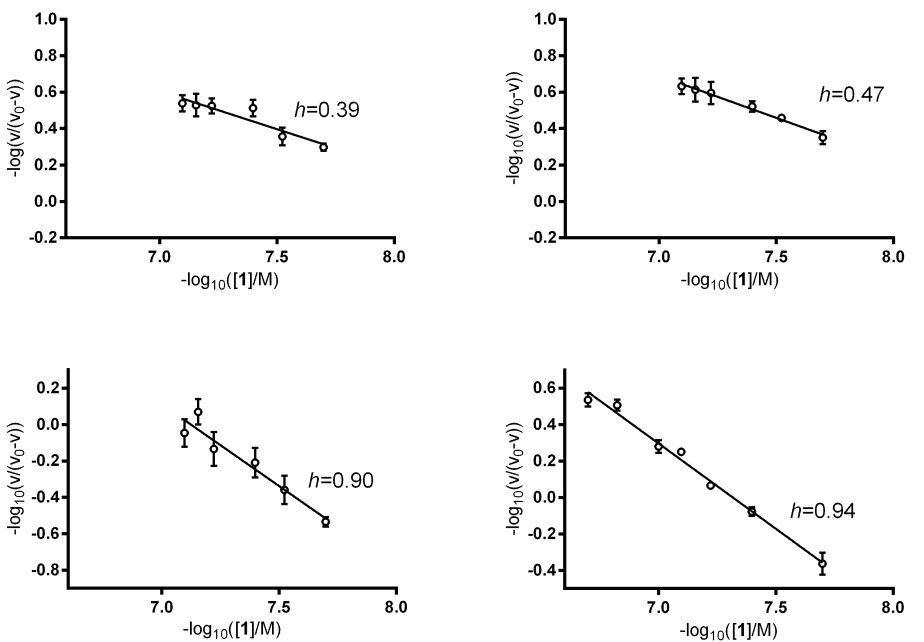

NQO1-G150S

No detectable activity

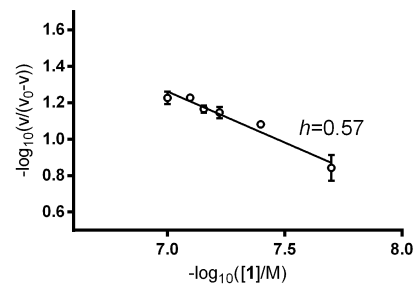

NQ01-G149S

NQ01-G174S

Figure 1. Human NQO1 showed negative cooperativity towards dicoumarol and related compounds. (a) Hill plots for NQO1 enzymatic activity in the presence of compounds 1-4. In this type of plot, $\log _{10}\left(\mathrm{~V} / \mathrm{v}_{0}-\mathrm{v}\right)$ is plotted against $\log _{10}\left[\right.$ Inhibitor], where $\mathrm{v}_{0}$ is the maximal or uninhibited rate. In such plots, the gradient of the line equals $-h$, such that positive cooperativity is indicated when $h>1.0$ and negative cooperativity when $h<1.0$. When $h=1.0$, there is no cooperativity. ${ }^{[25]}$ This method has been widely used to detect cooperative behaviour of enzymes towards their inhibitors (for example, to detect positive cooperativity in the inhibition of fructose 1,6-bisphosphatase by AMP ${ }^{[26]}$, to detect negative cooperativity in the fosmidomycin inhibition of 1-deoxy-D-xylulose-5-phosphate reductoisomerase, ${ }^{[27]}$ and to show the lack of cooperativity in the inhibition of alkaline phosphatase by L-tryptophan ${ }^{[28]}$ ). Each point represents the mean of three separate determinations, the error bars are standard deviations of these means and the lines are linear best fits to the data. Hill coefficients are given on the graphs. (b) Hill plots were constructed for the glycine to serine variants with $300 \mu \mathrm{M} \mathrm{NADH}, 70 \mu \mathrm{M}$ DCPIP and varying concentrations of dicoumarol. Reactions were measured

For internal use, please do not delete. Submitted_Manuscript 
at $37{ }^{\circ} \mathrm{C}$ using $5 \mathrm{nM}$ G149S-NQO1, $5 \mathrm{nM}$ and $8 \mathrm{nM}$ G150S-NQO1; the activity of G174S-NQO1 was too low to measure in the absence of additional FAD. These plots were used to estimate the Hill coefficient $(h)$. Each point represents the mean of three separate determinations and the error bars show the standard error of these means; triplicate Hill plots were constructed, one representative plot with a median value of $h$ shown. This was repeated in excess FAD for each enzyme (each enzyme stock was pre-diluted using FAD containing buffer such that it was 10x the concentration of active sites in the diluted stock; enzyme concentrations were decreased to account for the higher activity in the presence of FAD: G149S-NQO1, 2.5 nM; G150S-NQO1, 5 nM; G174S-NQO1, 10 nM).

Table 1: Kinetic and stability parameters for wild-type NQO1 and glycine to serine substituted variants.

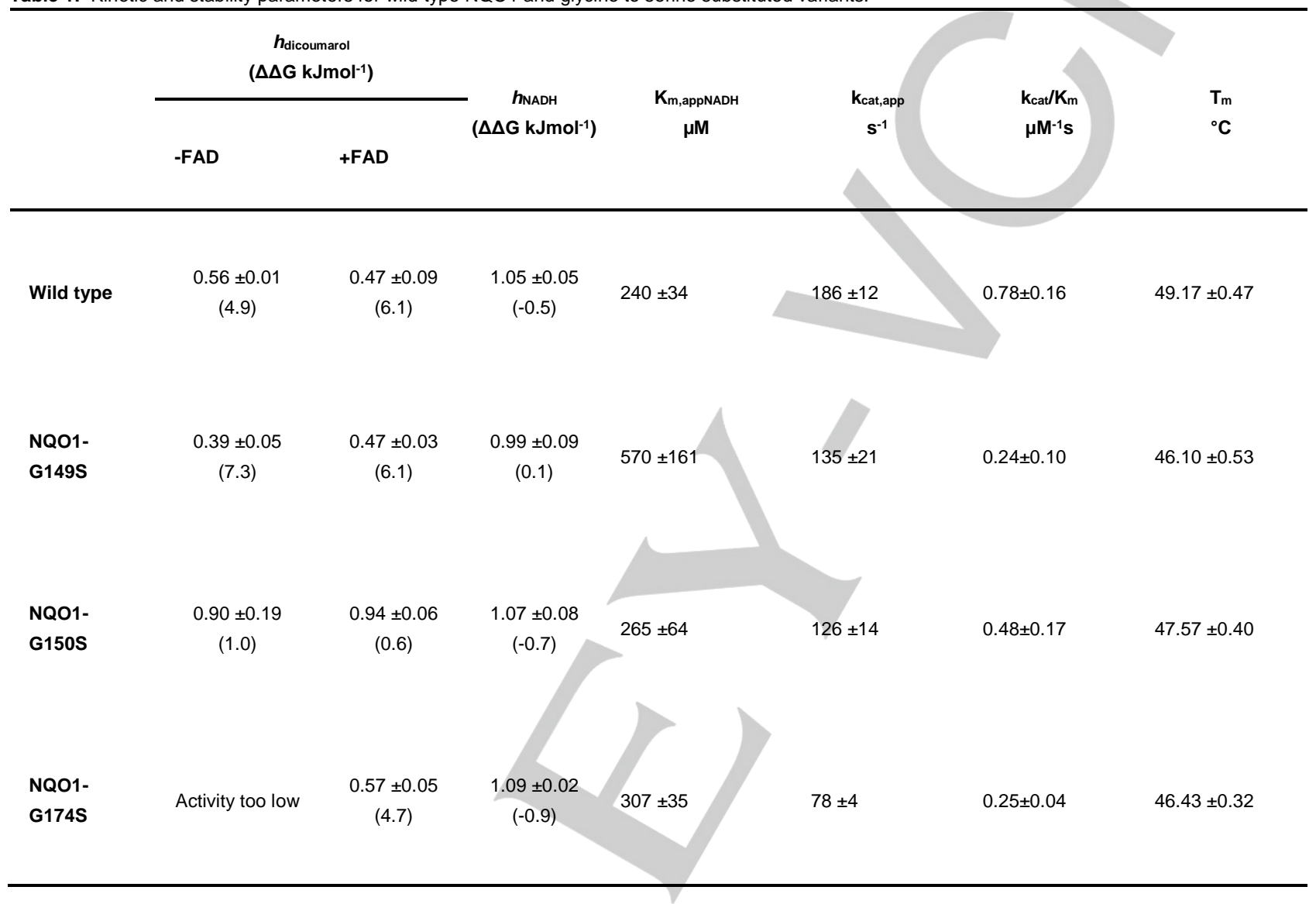

Kinetic measurements used wild type NQO1 (3 nM dimer), NQO1- G149S (3 nM dimer), NQO1-G150S (3 nM dimer) and NQO1-G174S (20 nM dimer) with $70 \mu \mathrm{MM}$ DCPIP at $37^{\circ} \mathrm{C}$. Non-enzymatic background rates were measured in triplicate at each concentration of NADH and $70 \mu M$ DCPIP at $37^{\circ} \mathrm{C}$ in $50 \mathrm{mM}$ phosphate buffer $\mathrm{pH} 7.4$ and the mean of these subtracted from the rates obtained from the linear section at the beginning of each progress curve with enzyme included (also in triplicate). Melting temperatures $\left(T_{m}\right)$ were determined (in triplicate) by DSF.

For internal use, please do not delete. Submitted_Manuscript 

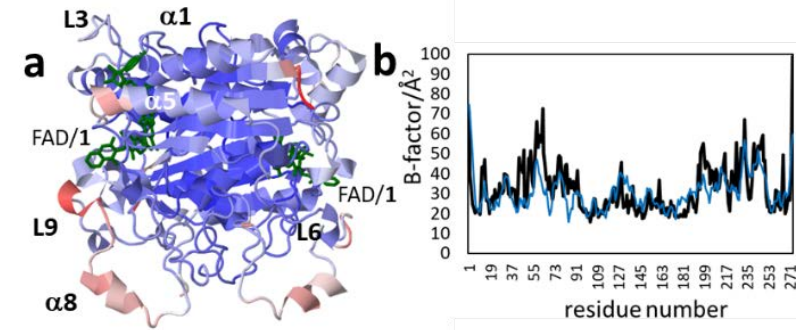

C
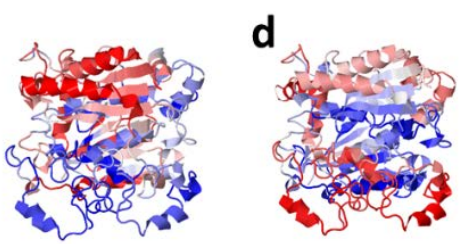

e

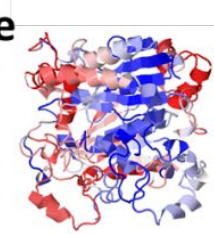

f

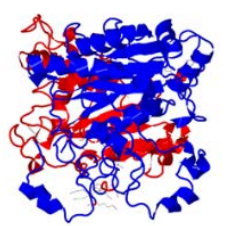

$\mathrm{h}$

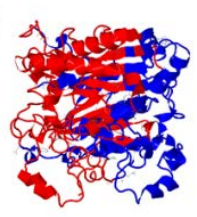

Figure 2: (a) Experimental B-factors for atoms of crystal structure of NQO1 (largest values in red), ${ }^{[22]} \mathrm{FAD}$ and ligand $\mathbf{1}$ in green; (b) Comparison of computed (black) and experimental (blue) B-factor values across NQO1 residues. Magnitude of motion for modes (c) $k=1$; (d) $k=2$; and (e) $k=3$ (red, most mobile; blue, least mobile). Dynamic domains for (f) $k=1$; (g) $k=2$; and (h) $k=3$. The domains are separated by the signs of eigenvectors and colored red $(+)$ and blue (-) respectively.

Using the Gaussian network model, we consider the predicted fluctuation profiles of the most global modes of motion for apo NQO1, with ligand 1 removed from both sites. We observe that these slowest frequency modes appear to represent strongly collective motions of NQO1: for the lowest frequency mode, labelled $k=1$, the higher amplitude motions of the protein (red regions in Figure $2 \mathrm{c}$ ) appear to belong to the NTD regions of the monomers. We note that blue region in Figure $2 \mathrm{c}$ indicates lower motion and defines a hinge region. For mode $k=2$, the mobility of NQO1 also involves CTD residues belonging to the $\alpha 8$ helices of both monomers (Figure $2 \mathrm{~d}$ ). For the $k=3$ mode, only one of the two $\alpha 8$ helices is engaged (Figure 2e). In terms of the direction of these motions, the $k=1$ and $k=2$ modes involve cross-monomer antisymmetric movement of NQO1 (Figure 2f,g) whereas for $k=3$, the monomers appear to librate separately in a wagging motion (Figure 2h). For all three modes, however, the interface for anticorrelated domain dynamics intersects with both NQO1 active sites (Figure 2f-h).

We may quantify the non-local nature of these soft modes by computing their collectivity, $\Omega_{k}$ (see Methods). ${ }^{[29]}$ In all cases, the modes are global motions, with collectivity values of $0.62,0.52$ and 0.46 for the respective $k=1, k=2$ and $k=3$ modes, ie. for the $k=1$ mode, $62 \%$ of the NQO1 residues are involved in these

modes respectively (Figure S8). The collectivity of other modes dimishes only slowly with increased frequency, eg. $\Omega_{k}$ has a value of 0.33 for mode $k=20$ (Figure S8).

Next, we considered the effect on the dynamics of NQO1 by introducing ligand 1 at one of protein's two active sites. For the three softest modes of the NQO1 complex, the magnitude and direction of the motions remain qualitatively similar to that of the apo protein modes shown in Figure 2c-h. Nevertheless, there are differences in the detailed motion of the protein predicted on ligand association: as perhaps might be expected, there is a significant decrease in mode motion of active site residues in the vicinity of the bound ligand (Figure 3a); for example, for $k=1$, this is most notable for residues 115 - 150; this region includes active site residue Tyr 128 , the mobility of which drops by $62 \%$ on ligand binding (Figure 3a).

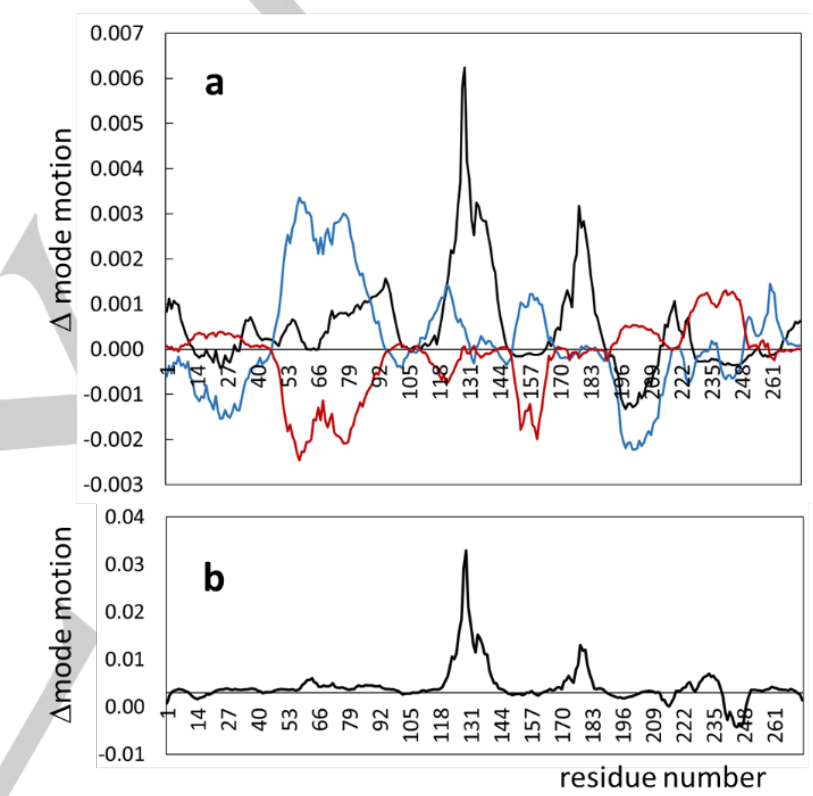

Figure 3. (a) Difference in magnitudes of motion on ligand 1 binding (computed as apo - complexed), for modes $k=1$ (black), 2 (blue) and 3 (red); (b) Difference in mode motion on ligand $\mathbf{1}$ binding of summed ten slowest modes of motion.

By contrast, in some parts of the enzyme, there is an increase in mode motion on ligand binding. For $k=1$, this occurs mainly at NTD helix $\alpha 5$ that traverses NQO1 (residues $196-210$, Figure $3 a$ ); the spatial location of $\alpha 5$ is shown in Figure $2 a$. For $k=2$, increasing mobility on ligand association is found at helices $\alpha 1$ (residues $11-44$ ) and again at $\alpha 5$ (Figures $2 a$ and 3a). Finally, for the $k=3$ mode, residues $51-91$ and $150-164$ are more mobile on complexation (Figure 3a), The first region includes loop L3 (Figure 2a) which is highlighted as a flexible region from Bfactors (Figure $2 b$ ) and from previous work. ${ }^{[6 a, 6 c]}$ Interestingly, residues 15 - 164 correspond to loop L6 in the second, unoccupied binding site (Figure 2a). These amino acids are remote to the binding site, although we note there is an equal and opposite effect from mode 2. Overall, considering the cumulative contribution of the 10 slowest modes (Figure $3 b$ ), on binding of $\mathbf{1}$, the motion is damped around the ligand binding site and a

For internal use, please do not delete. Submitted_Manuscript 
neighbouring shell of residues centred around Gly180 and Phe181. By contrast, there is an increase in flexibility around amino acids $242-251$, i.e. helix $\alpha 8$ in the CTD.

To further probe the sensitivity of amino acids in NQO1 to receiving and propagating mechanical signals, we applied perturbation-response scanning (PRS) to the Gaussian network model of the protein. ${ }^{[30]}$ From PRS analysis, a range of residues across NQO1 appear to be sensitive to perturbation (black, Figure 4 ), with peaks in signal profile at secondary structure elements $\alpha 1$, $\alpha 7, \beta 4-\mathrm{L} 6$ and $\alpha 5$ in the NTD and $\alpha 8$ in the CTD. This pattern is broadly reflected by the profile of residues which are effective in propagating signal through the protein (red, Figure 4). Clearly, NQO1 forms a highly collective dynamic network of amino acids, capable of responding to ligand binding and communicating that response through the protein.

Specifically, PRS suggests that the $\alpha 8$ helix, residues 242 251 of the CTD, would respond (Figure 4) to the increase in mode motion on ligand binding (Figure $3 b$ ) and would be capable of propagating this signal remotely throughout the protein, including to regions such as loop L6 near the second binding site, potentially impairing binding of a second ligand at that site.

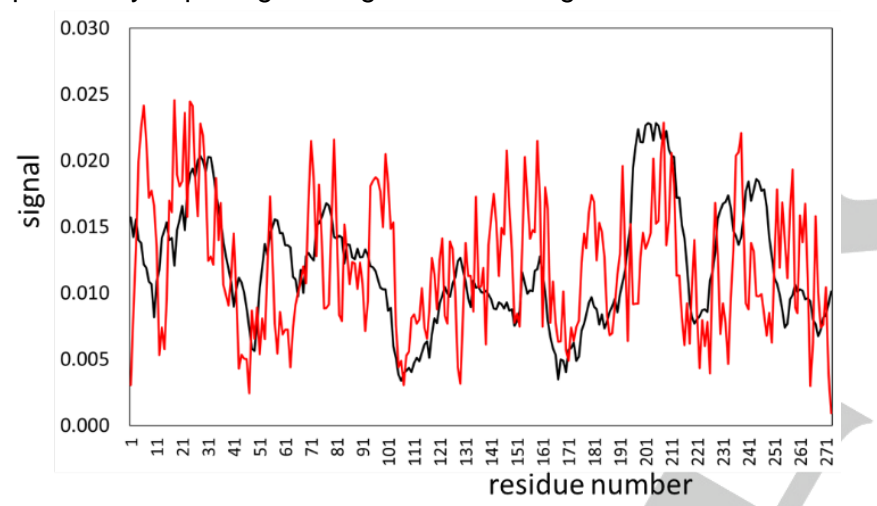

Figure 4. Magnitude of perturbation (black) and response (red) signal of receiver and propagator residues respectively in apo NQO1 model.

Given this highly interconnected network of residues in NQO1, sensitive to ligand binding, it is perhaps surprising that only the G150S variant led to abrogation of negative cooperativity, whereas G149S and G174S variants did not (Table 1). The slow modes of NQO1 computed from the Gaussian network model indicate that residues Gly149 and Gly150, and to a lesser extent, Gly174, lie in regions of near-zero mode motion (Figure 3b); residues with low motion have been shown in elastic network studies of other proteins to point towards significant hinge or functional residues within a macromolecule..$^{[31]}$ Indeed, all three glycines lie near the ligand binding site (Figure 5a) and the backbones of Gly149 and Gly150 play a specific role in hydrogen bonding with the isoalloxazine ring of FAD. However, from inspection of the NQO1/1 crystal structure, we note that perturbation to serine of Gly150, but not Gly149 or Gly174, would directly obstruct the substrate/inhibitor binding site (Figure $5 b$ ). This in turn could lead to displacement of immediately neighboring residues (Tyr126, Tyr128), second shell residues (Gly180, Phe181) and beyond this, the CTD helix $\alpha 8$ residues $242-251$
(Figure 5b), interfering with the negatively cooperative communication between active sites.
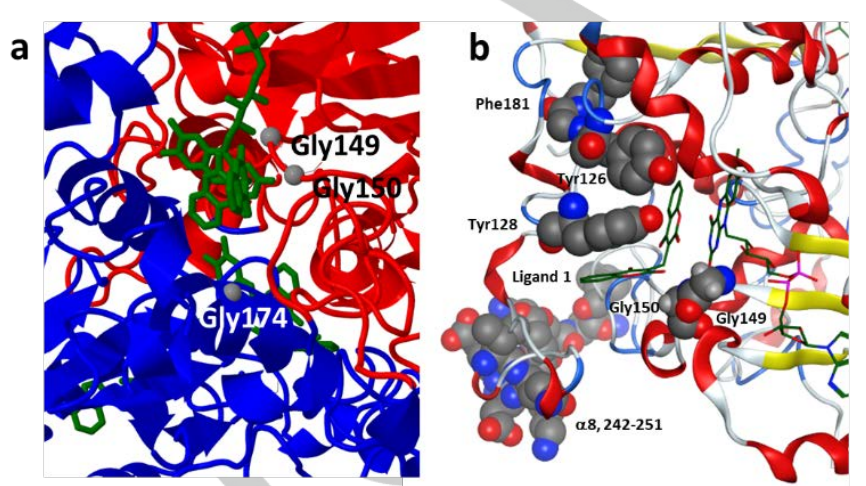

Figure 5. (a) Location of Gly149, Gly150 and Gly174 at active site of NQO1 (monomers coloured red and blue; FAD and 1 in green). (b) Spatial relationship of Gly149 and Gly150 to promixal residues linked to inter-active site communication.

\section{Conclusions}

These findings have considerable implications for the function of the protein in vivo and its exploitation as an anti-cancer drug target. Our studies imply that, in addition to adopting a correctly folded three-dimensional structure, NQO1 displays highly collective inter-domain and inter-monomer dynamics. This coupled network of amino acids is sensitive to ligand binding and can be disrupted by a single amino acid change, with functional consequences for the protein. Therefore, it is possible that the cancer-associated polymorphisms exert their pathogenic effect, in part, through alteration of the dynamics of the protein. This hypothesis is supported by recent studies demonstrating that the P187S variant of NQO1 is more likely to populate partially unfolded states. ${ }^{[6 a, 6 c, 16 a-c]}$ This mutation site is found at the monomer-monomer interface and was able to interfere remotely with the structure and dynamics of the FAD binding site, loop L3 and the CTD in particular, again suggesting a strongly coupled dynamical communication network within NQO1.

There are also implications for cells expressing wild-type NQO1. Moscovitz et al. have raised the intriguing notion that NQO1-mediated protein stability may be linked to cellular metabolic status: in particular a regulatory role for cellular FAD has been proposed. ${ }^{[32]}$ In addition, the ability of NQO1 to prevent degradation of proteins via the $20 \mathrm{~S}$ proteosome is NADHdependent which also suggests an underlying redox sensing mechanism for the chaperone function of NQO1. ${ }^{[10 a]}$ Coumarinbased inhibitors such as dicoumarol (1) compete with NADH for binding to NQO1 and thereby target p53 for degradation. Although the precise mechanism for this is unclear, it is likely to involve direct interactions between NQO1 and p53.[33] Negative cooperativity occurs only with inhibitors and not with $\mathrm{NAD}(\mathrm{P}) \mathrm{H}$. One consequence of negative cooperativity is to dampen the responsiveness of a system. Some enzymes involved in

For internal use, please do not delete. Submitted_Manuscript 
signalling exhibit negative cooperativity and, consequently, are switched on over a wide range of substrate concentrations. ${ }^{[34]}$ It is possible that a similar situation occurs here: NQO1's negatively cooperative response to inhibitors may result in inhibition of the p53 interaction over a wide range of concentrations. Therefore, the most pharmacologically useful NQO1 inhibitors may be those which maximise both affinity and negative cooperativity.

It is interesting to speculate on the evolutionary significance of negative cooperativity in NQO1. To date, no cellular metabolites have been identified which inhibit NQO1 in vivo. All known inhibitors are either exogenous drugs or toxins. However, the existence of strong negative cooperativity in this enzyme suggests that it confers some selective advantage. It may be important in the mechanism of protein chaperoning, as noted above. Another possibility is that the negative cooperativity in FAD binding coincidentally affects interaction with inhibitors such as dicoumarol. ${ }^{[7]}$ If this was the case, it seems likely that the negative cooperativity is important in the protein's redox sensing role potentially enabling it to act over a wide range of cellular FAD concentrations.

\section{Experimental Section}

Reagents, chemicals and NQO1 inhibitors: Unless otherwise stated, all reagents and chemicals were supplied by Sigma Aldrich (Poole, Dorset, UK). Dicoumarol was dissolved to a concentration of $25 \mathrm{mM}$ in fresh 0.13 $\mathrm{M}$ sodium hydroxide. Etoposide was dissolved to a concentration of $10 \mathrm{mM}$ in DMSO. The NQO1 inhibitors (Scheme 1) were dissolved in DMSO to give a final concentration of $25 \mathrm{mM}$. Compounds $2-4$ were prepared as described previously. ${ }^{[14 a]}$

Cell lines and cell culture: Cells were maintained at $37^{\circ} \mathrm{C}$ in exponential phase in a humidified incubator with $5 \% \mathrm{CO}_{2}$. $\mathrm{HCT} 116$ colon carcinoma cells were grown in Dulbecco's Modified Eagle Medium (DMEM) supplemented with $10 \%(\mathrm{v} / \mathrm{v})$ heat-inactivated foetal calf serum and $2 \mathrm{mM}$ L-glutamine. MCF10A breast epithelial cells were maintained in DMEM/F12 (1:1) containing L-glutamine (2 mM), horse serum (5\%), epidermal growth factor $(20 \mu \mathrm{g} / \mathrm{mL})$, hydrocortisone $(0.5 \mu \mathrm{g} / \mathrm{mL})$, cholera toxin $(0.1$ $\mu \mathrm{g} / \mathrm{mL})$, insulin $(10 \mu \mathrm{g} / \mathrm{mL})$ and penicillin/streptomycin (1\%). Human umbilical vein endothelial cells (HUVECs) were obtained from TCS CellWorks (Buckingham, UK) and cultured as per the supplier's instructions. Cells were used between passages 2-16 (HCT116 and MCF10A) and 2-8 (HUVECs).

Western blot analysis of p53 levels: Exponentially growing cells were seeded at $10^{5}$ cells/well in six well plates, left to adhere overnight, then treated with or without $10 \mu \mathrm{M}$ etoposide for $24 \mathrm{~h}$ prior to treatment with 50 , 100,200 or $400 \mu \mathrm{M}$ of each inhibitor and incubated for four hours. Cells were harvested by scraping into lysis buffer ( $50 \mathrm{mM}$ Tris- $\mathrm{HCl}(\mathrm{pH} 7.4), 120$ $\mathrm{mM} \mathrm{NaCl}, 5 \mathrm{mM}$ EDTA, 0.5\% v/v Nonidet P-40, 1 mM dithiothreitol, $1 \mathrm{mM}$ phenylmethanesulphonyl fluoride, $2 \mathrm{mM}$ sodium orthovanadate, $2 \mathrm{mM}$ sodium fluoride, $20 \mathrm{mM}$ ß-glycerolphosphate, $5 \mathrm{mM}$ sodium pyrophosphate, and $1 \mathrm{mg} / \mathrm{ml}$ Protease Inhibitor Cocktail tablet (Roche, UK). Samples were then sonicated on ice and the protein concentration of each was estimated using the BCA assay. For each sample, $40 \mu \mathrm{g}$ protein was loaded and electrophoresed. p53 antibody (DO-1, Insight Biotechnology, Middlesex, UK) was used to assess p53 protein levels, while $\beta$-actin or tubulin antibodies were used as loading controls. Primary antibodies were then detected using the appropriate HRP-conjugated secondary antibody and bands were visualized using the ECL detection system (GE Healthcare, Buckinghamshire, UK). Exponentially growing HUVECs were seeded at $\left(2.5 \times 10^{5}\right.$ cells/well) in six well plates and left to adhere overnight, before exposure to 10 Gy ionizing radiation ( $250 \mathrm{kV} \mathrm{X-}$ rays). The cells were then incubated for $4 \mathrm{~h}$ with and without the inhibitor before being harvested and probed for $\mathrm{p} 53$ as described above.

Measurement of NQO1 kinetics and inhibition: Recombinant human NQO1 was either a gift from Professor David Ross and Dr David Siegel or prepared as described previously ${ }^{[16 a]}$ Reduction of dichlorophenolindophenol (DCPIP) by NADH was measured spectrophotometrically at $600 \mathrm{~nm}$ at $37{ }^{\circ} \mathrm{C}$ in $50 \mathrm{mM}$ sodium phosphate buffer (pH 7.4). For measurement of wild-type NQO1 with $\mathrm{NAD}(\mathrm{P}) \mathrm{H}$ and inhibition of wild-type NQO1 by 1-4 in the presence of $200 \mu \mathrm{M}$ NADH a Cary 100 Scan UV-visible spectrophotometer was used with $40 \mu$ M DCPIP and $40 \mu \mathrm{M} \mathrm{NQO1}$ in a total reaction volume of $900 \mu \mathrm{l}$. For inhibition of glycine to serine variant forms of NQO1 by 1 a Multiskan Spectrum platereader (Thermo Scientific) was used with $300 \mu \mathrm{M} N A D H, 70 \mu \mathrm{M}$ DCPIP, 3-20 nM NQO1 and $0.9 \mu \mathrm{M}$ lysozyme in a total volume of 200 $\mu \mathrm{l} .{ }^{[16 a]}$ Reactions were initiated by the addition of NQO1 and rates were measured for $2 \mathrm{~min}$ at $37^{\circ} \mathrm{C}$. Data were fitted to the Michaelis-Menten and Hill equations ${ }^{[35]}$ using GraphPad Prism 5.0.

For inhibition experiments, compounds 1-4 were dissolved in $\mathrm{DMSO} / 0.13 \mathrm{M} \mathrm{NaOH}$ and diluted such that the final concentration of these solvents was $0.5 \%(\mathrm{v} / \mathrm{v})$. Inhibition data were analysed by Hill plots in which $\log _{10}\left(\mathrm{v} / \mathrm{v}_{0}-\mathrm{v}\right)$ is plotted against $\log _{10}\left[\right.$ Inhibitor], where $\mathrm{v}_{0}$ is the rate of the uninhibited reaction. In such plots, the gradient of the line equals $-h$, such that positive cooperativity is indicated when $h>1.0$ and negative cooperativity when $h<1.0^{[25 a, 35]}$ Cooperativity was also assessed using the method of Forsén and Linse. ${ }^{[36]}$ This method enable the estimation of difference in free energy of binding between the high and low affinity states of the protein $(\Delta \Delta G)$. It makes a number of assumptions. First that cooperativity can be represented by two equilibria: $K_{1}$, the dissociation constant for the ligand and the unliganded enzyme and $\mathrm{K}_{2}$, the dissociation constant for the ligand and enzyme already bound to one ligand molecule (i.e. these are "macroscopic" binding constants which reflect the overall ligand occupancy of the protein). In the case of negative cooperativity, $\mathrm{K}_{2}$ will be greater than (i.e. lower affinity) than $K_{1}$. Second, that in the unliganded enzyme, the affinities of the two binding sites for the inhibitor are equal (i.e. that the "microscopic" binding constants for the two active sites are the same). This would be expected in a homodimeric enzyme like NQO1. However, it should be noted that there is no positive evidence in favour of this assumption. Thus, the $\Delta \Delta \mathrm{G}$ values reported here should be seen as minimum values.

The Hill coefficient is related to these two equilibrium constants by the equation:

$$
h=\frac{4 \sqrt{K_{2} / K_{1}}}{1+2 \sqrt{K_{2} / K_{1}}}
$$

The difference in free energies can be calculated according to the equation:

$$
\Delta \Delta G=-R T \log _{e} \frac{4 K_{2}}{K_{1}}
$$


Where, $\mathrm{R}$ is the universal gas constant and $\mathrm{T}$ is the absolute (Kelvin) temperature.

Ultracentrifugation: NQO1 was buffer exchanged and further purified on a Superdex 200 10/30 gel filtration column (GE-Lifesciences) in PBS. Peak fractions were pooled and diluted in buffer with or without the addition of inhibitors to a final concentration $15 \mu \mathrm{M}$. Samples were then loaded into a two-sector Epon filled centerpiece covered with quartz glass windows and centrifuged at $45000 \mathrm{rpm}$ at $20^{\circ} \mathrm{C}$. Sedimentation was monitored every 90 s using absorbance at $450 \mathrm{~nm}$ until full sedimentation had been reached. The data were analysed using Sedfit software. ${ }^{[37]}$

Isothermal titration calorimetry of ligand binding: Ligand binding to NQO1 was studied at $25^{\circ} \mathrm{C}$ using a VP-ITC microcalorimeter (Microcal Inc, MA). Data acquisition and analysis were carried out using ORIGIN 7.0 (Microcal Inc., MA). Ligand binding was quantified by fitting the evolved heat per injection to the standard Wiseman equation. Fitting of the binding isotherm was carried out through multiple iterations until a minimum $\chi^{2}$ value was obtained. Reported values are the average of at least three runs. NQO1 monomer concentration was chosen so as to give $c$ values in the 100-300 range (typically 3-5 $\mu \mathrm{M}$ ). Both NQO1 and ligands were dissolved in $50 \mathrm{mM}$ phosphate $\mathrm{pH} 7.5$ buffer containing $5 \mathrm{mM}$ FAD and $2.5 \%(\mathrm{v} / \mathrm{v})$ DMSO.

NQO1 protein stability: Differential scanning fluorimetry was performed as described previously, taking advantage of the increase in the natural fluorescence of the FAD molecules which occurs when the cofactor is released as the protein denatures. ${ }^{[18,38]}$ Limited proteolysis with increasing concentrations of chymotrypsin $(0,5,10,35,60,90,360,630 \mathrm{nM})$ was performed with wild-type and variant NQO1 protein (10 $\mu \mathrm{M}$ dimer) which had been pre-incubated at $37^{\circ} \mathrm{C}$ for $15 \mathrm{~min}$. The reaction was allowed to proceed for $30 \mathrm{~min}$ after which time it was stopped by addition of an equal volume of tris-tricine SDS loading buffer (12\% (w/v) SDS; $6 \%(\mathrm{w} / \mathrm{v})$ DTT; $30 \%$ (v/v) glycerol; $150 \mathrm{mM}$ Tris/ $\mathrm{HCl} \mathrm{pH}$ 7). Reactions were analysed using tris-tricine SDS PAGE. ${ }^{[39]}$

Elastic network modelling: For generating the GNM models, the coordinates of the crystal structure of NQO1 bound to ligands 1 were taken from $\mathrm{PDB}$ code $2 \mathrm{~F} \mathrm{O}^{[22]}$ using chains $\mathrm{B}$ and $\mathrm{D}$. FAD was modelled by springs centred on atoms $\mathrm{C} 7, \mathrm{~N} 5, \mathrm{~N} 3, \mathrm{C} 1$ ', C4', P, PA, C4b and N9a. The ligand was modelled by atoms $\mathrm{C} 5, \mathrm{C} 1$ and $\mathrm{C} 15$. The $\mathrm{C}_{\alpha}$ atoms of the amino acids, and these ligand atoms where relevant, were used to build the Kirchhoff connectivity matrices $\Gamma$ using a cutoff $r_{c}$ of $7.3 \AA$. For $N$ residues in a molecular system, diagonalization of $\Gamma$ provides $N$-1 modes; the resulting modes $k$ of eigenvector $\mathbf{u}_{k}$ with the smallest eigenvalue $\lambda_{k}$ correspond to softest and most cooperative motions within the molecular system. Contribution to motion of mode $k$ is computed as $\left\langle\Delta \boldsymbol{R}_{\boldsymbol{i}} \cdot \Delta \boldsymbol{R}_{j}\right\rangle_{\boldsymbol{k}}=$ $\left(3 \boldsymbol{k}_{B} \boldsymbol{T} / \gamma\right)\left[\lambda_{k}^{-1} \boldsymbol{u}_{k} \boldsymbol{u}_{k}^{T}\right]_{i j}$ where $\Delta \boldsymbol{R}_{i}$ is the Gaussian fluctuation in position of residue $i, \boldsymbol{k}_{B}$ is Boltzmann's constant, $T$ is temperature and $\gamma$ is the network force constant. Differences in mode motion are computed as the square displacement of residues driven by a given mode of complexed NQO1 subtracted from that of apo NQO1. Following Brüschweiler, ${ }^{[29]}$ the level of collectivity, $\boldsymbol{\Omega}_{\boldsymbol{k}}$, for a mode $k$ is defined as $\boldsymbol{\Omega}_{\boldsymbol{k}}=(\mathbf{1}$ ) $\boldsymbol{N}) \exp \left\{-\sum_{i} \boldsymbol{u}_{\boldsymbol{k}, i}^{2} \boldsymbol{l n}\left[\boldsymbol{u}_{\boldsymbol{k}, i}^{2}\right]\right\}$ for $N$ residues $i$. GNM analysis was performed using the iGNM 2.0 conformational dynamics interface and database. ${ }^{[30 b]}$ Perturbation response scanning ${ }^{[31]}$ is a method to compute the influence/sensitivity that each residue has on/to every other residue. Based on linear response theory, PRS is achieved by exerting a force of a given magnitude on the network, one residue at a time, and observe the response of the overall network. PRS30 was used to identify the most sensitive residues of NQO1.

\section{Acknowledgements}

We are indebted to Professor David Ross and Dr David Siegel (University of Colorado, USA) for a supply of recombinant human NQO1. We would also like to thank Dr Sharon Sneddon and Miss Catherine Treslove (University of Manchester, UK) for carrying out the p53 analyses in the normal cells and Prof Aaron Maule (Queen's University, Belfast, UK) for access to equipment. RAB thanks Mark Dunstan for helpful discussions. This work was supported by grants from the Medical Research Council, G0500366 (to IJS) and the Association for International Cancer Research, 08-0152 (to IJS, KAN, RAB and RCW). CFM thanks the Department of Employment and Learning, Northern Ireland (DELNI) for a PhD studentship.

Keywords: Quinone oxidoreductase • Protein flexibility • Elastic network model $\bullet$ Enzyme cooperativity $\bullet$ Cancer-associated protein

[1] a) R. Li, M. A. Bianchet, P. Talalay, L. M. Amzel, Proc Natl Acad Sci USA 1995, 92, 8846-8850; b) S. K. Beaver, N. Mesa-Torres, A. L. Pey, D. J. Timson, Biochim Biophys Acta 2019, 1867, 663-676.

[2] C. Lind, P. Hochstein, L. Ernster, Arch Biochem Biophys 1982, 216, 178-185.

[3] C. Lind, E. Cadenas, P. Hochstein, L. Ernster, Methods Enzymol 1990, 186, 287-301.

[4] S. Hosoda, W. Nakamura, K. Hayashi, J Biol Chem 1974, 249 6416-6423.

[5] B. Rase, T. Bartfai, L. Ernster, Arch Biochem Biophys 1976, 172, 380-386.

[6] a) E. Medina-Carmona , R. J. Palomino-Morales, J. E. Fuchs, P. G. Esperanza, M. T. Noel, E. Salido, D. J. Timson, A. L. Pey, Sci Rep 2016, 6, 20331; b) E. Medina-Carmona, J. E. Fuchs, J. A. Gavira, N. Mesa-Torres, J. L. Neira, E. Salido, R. Palomino-Morales, M. Burgos, D. J. Timson, A. L. Pey, Hum Mol Genet 2017, 26, 3531-3544; c) E. Medina-Carmona, J. L. Neira, E. Salido, J. E. Fuchs, R. Palomino-Morales, D. J. Timson, A. L. Pey, Sci Rep 2017, 7, 44532.

[7] R. Claveria-Gimeno, A. Velazquez-Campoy, A. L. Pey, Arch Biochem Biophys 2017, 636, 17-27.

[8] A. L. Pey, C. F. Megarity, D. J. Timson, Biosci Rep 2019, 39, BSR20180459.

[9] a) G. Asher, Z. Bercovich, P. Tsvetkov, Y. Shaul, C. Kahana, Mol Cell 2005, 17, 645-655; b) G. Asher, J. Lotem, R. Kama L. Sachs, Y. Shaul, Proc Natl Acad Sci USA 2002, 99, 30993104; cG. Asher, P. Tsvetkov, C. Kahana, Y. Shaul, Genes Dev 2005, 19, 316-321.

[10] a) G. Asher, J. Lotem, B. Cohen, L. Sachs, Y. Shaul, Proc Natl Acad Sci USA 2001, 98, 1188-1193; b) P. Tsvetkov, G. Asher, V. Reiss, Y. Shaul, L. Sachs, J. Lotem, Proc Natl Acad Sci USA 2005, 102, 5535-5540; c) D. Thapa, P. Meng, R. G. Bedolla, R. L. Reddick, A. P. Kumar, R. Ghosh, Cancer Res 2014, 74, 5644-5655.

[11] a) M. Belinsky, A. K. Jaiswal, Cancer Metastasis Rev 1993 12, 103-117; b) M. A. Colucci, C. J. Moody, G. D. Couch, Org Biomol Chem 2008, 6, 637-656.

[12] A. Lewis, M. Ough, L. Li, M. M. Hinkhouse, J. M. Ritchie, D. R. Spitz, J. J. Cullen, Clin Cancer Res 2004, 10, 4550-4558.

For internal use, please do not delete. Submitted_Manuscript 
a) C. Laruelle, J. J. Godfroid, J Med Chem 1975, 18, 85-90; b) G. A. James, J Clin Path 1949, 2, 45-48; c) R. L. Macmillan, Science 1948, 108, 416-417; d) D. J. Timson, Curr Drug Targets 2017, 18, 500-510.

[14] a) K. A. Nolan, K. A. Scott, J. Barnes, J. Doncaster, R. C Whitehead, I. J. Stratford, Biochem Pharmacol 2010, 80, 977981; b) K. A. Nolan, H. Zhao, P. F. Faulder, A. D. Frenkel, D. J. Timson, D. Siegel, D. Ross, T. R. Burke Jr, I. J Stratford, R. A. Bryce, J Med Chem 2007, 50, 6316-6325; c) K. A. Scott, J. Barnes, R. C. Whitehead, I. J. Stratford, K. A. Nolan, Biochem Pharmacol 2011, 81, 355-363; d) J. Bian, B. Deng, L. Xu, X. Xu, N. Wang, T. Hu, Z. Yao, J. Du, L. Yang, Y. Lei, X. Li, H. Sun, X. Zhang, Q. You, Eur J Med Chem 2014, 82, 56-67; e) C. Lopez-Lira, J. H. Alzate-Morales, M. Paulino, J. MellaRaipan, C. O. Salas, R. A. Tapia, J. Soto-Delgado, Chem Biol Drug Des 2018, 91, 29-38; f) D. L. Dehn, D. Siegel, K. S. Zafar, P. Reigan, E. Swann, C. J. Moody, D. Ross, Mol Cancer Ther 2006, 5, 1702-1709; g) M. Faig, M. A. Bianchet, S. Winski, R. Hargreaves, C. J. Moody, A. R. Hudnott, D. Ross, L. M. Amzel, Structure 2001, 9, 659-667; h) P. Reigan, M. A. Colucci, D. Siegel, A. Chilloux, C. J. Moody, D. Ross, Biochemistry 2007, 46, 5941-5950; i) S. L. Winski, M. Faig, M. A. Bianchet, D. Siegel, E. Swann, K. Fung, M. W. Duncan, C. J. Moody, L. M. Amzel, D. Ross, Biochemistry 2001, 40, 15135-15142.

[15] B. Lajin, A. Alachkar, Brit J Cancer 2013, 109, 1325-1337.

[16] a) A. L. Pey, C. F. Megarity, D. J. Timson, Biochim Biophys Acta 2014, 1842, 2163-2173; b) I. G. Munoz, B. Morel, E. Medina-Carmona, A. L. Pey, FEBS Lett 2017, 591, 28262835 ; c) W. D. Lienhart, V. Gudipati, M. K. Uhl, A. Binter, S. A Pulido, R. Saf, K. Zangger, K. Gruber, P. Macheroux, FEBS J. 2014, 281, 4691-4704; d) R. D. Traver, D. Siegel, H. D. Beall, R. M. Phillips, N. W. Gibson, W. A. Franklin, D. Ross, Brit J Cancer 1997, 75, 69-75; e) D. Siegel, S. M. McGuinness, S. L. Winski, D. Ross, Pharmacogenetics 1999, 9, 113-121. K. A. Nolan, J. R. Doncaster, M. S. Dunstan, K. A. Scott, A. D. Frenkel, D. Siegel, D. Ross, J. Barnes, C. Levy, D. Leys, R. C. Whitehead, I. J. Stratford, R. A. Bryce, J Med Chem 2009, 52, 7142-7156.

a) C. F. Megarity, J. R. Gill, M. C. Caraher, I. J. Stratford, K. A. Nolan, D. J. Timson, FEBS Letts 2014, 588, 1666-1672; b) C. F. Megarity, H. K. Looi, D. J. Timson, FEMS Yeast Res 2014, 14, 797-807.
L. Ernster, L. Danielson, M. Ljunggren, Biochim Biophys Acta 1962, 58, 171-188.

N. M. Goodey, S. J. Benkovic, Nat Chem Biol 2008, 4, 474482.

F. Huang, W. M. Nau, Angew Chem Int Ed 2003, 42, 22692272.

G. Asher, O. Dym, P. Tsvetkov, J. Adler, Y. Shaul, Biochemistry 2006, 45, 6372-6378.

a) T. Haliloglu, I. Bahar, B. Erman, Phys Rev Letts 1997, 79, 3090; b) I. Bahar, A. R. Atilgan, B. Erman, Fold Des 1997, 2, 173-181.

a) I. Bahar, J Gen Physiol 2010, 135, 563-573; b) C. Chennubhotla, Z. Yang, I. Bahar, Mol Biosyst 2008, 4, 287292.

a) P. C. Engel, in Enzymology LabFax, Bio Scientific Publishers, Oxford, UK, 1996; b) H. Abeliovich, Biophys J 2005, 89, 76-79.

K. Takata, B. M. Pogell, J Biol Chem 1965, 240, 651-662. L. Mercklé, A. de Andrés-Gómez, B. Dick, R. J. Cox, C. R. Godfrey, Chembiochem 2005, 6, 1866-1874.

C. W. Lin, H. G. Sie, W. H. Fishman, The Biochem J 1971, 124, 509-516.

R. Brüschweiler, J Chem Phys1995, 102, 3396-3403.

a) C. Atilgan, A. R. Atilgan, PLoS Comput Biol 2009, 5, e1000544; b) H. Li, Y. Y. Chang, L. W. Yang, I. Bahar, Nucleic Acids Res 2016, 44, D415-D422.

L. W. Yang, I. Bahar, Structure 2005, 13, 893-904.

O. Moscovitz, P. Tsvetkov, N. Hazan, I. Michaelevski, H. Keisar, G. Ben-Nissan, Y. Shaul, M. Sharon, Mol Cell 2012, 47, 76-86.

A. Anwar, D. Dehn, D. Siegel, J. K. Kepa, L. J. Tang, J. A Pietenpol, D. Ross, J Biol Chem 2003, 278, 10368-10373. J. E. Ferrell, Jr., J Biol 2009, 8, 53.

a) A. V. Hill, J Physiol 1910, 40, 4-7; b) D. J. Timson, Curr Enzym Inhib 2015, 11, 12-31.

a) S. Forsen, S. Linse, Trends Biochem Sci 1995, 20, 495497; b) S. Linse, A. Helmersson, S. Forsen, J Biol Chem 1991, 266, 8050-8054.

P. Schuck, Biophys J 2000, 78, 1606-1619.

F. Forneris, R. Orru, D. Bonivento, L. R. Chiarelli, A. Mattevi, FEBS J 2009, 276, 2833-2840.

H. Schagger, Nat Protoc 2006, 1, 16-22. 
WILEY-VCH

\section{Entry for the Table of Contents}

\section{FULL PAPER}

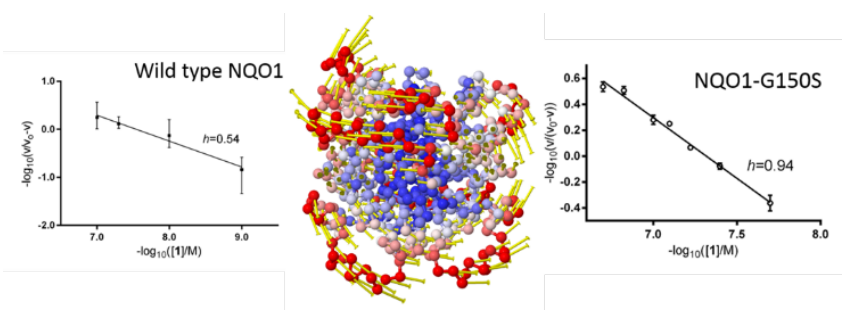

Clare F. Megarity, Hoda Abdel-Aal Bettley, M. Clare Caraher, Katherine A. Scott, Roger C. Whitehead, Thomas A. Jowitt, Aldo Gutierrez, Richard A. Bryce, Karen A. Nolan, Ian J. Stratford, David J. Timson*

\section{Page No. - Page No.}

Negative cooperativity in NAD(P)H quinone oxidoreductase 1 (NQO1)

\section{Table of contents text:}

NQO1 is a flavoprotein which catalyses the reduction of quinones and related compounds. The enzyme demonstrates negative cooperativity towards a competitive inhibitor, dicoumarol. A Gaussian network model, suggests that this arises from a high degree of collective motion within the protein. These findings have implications for understanding NQO1's cellular roles and in the discovery of anticancer drugs which target the enzyme.

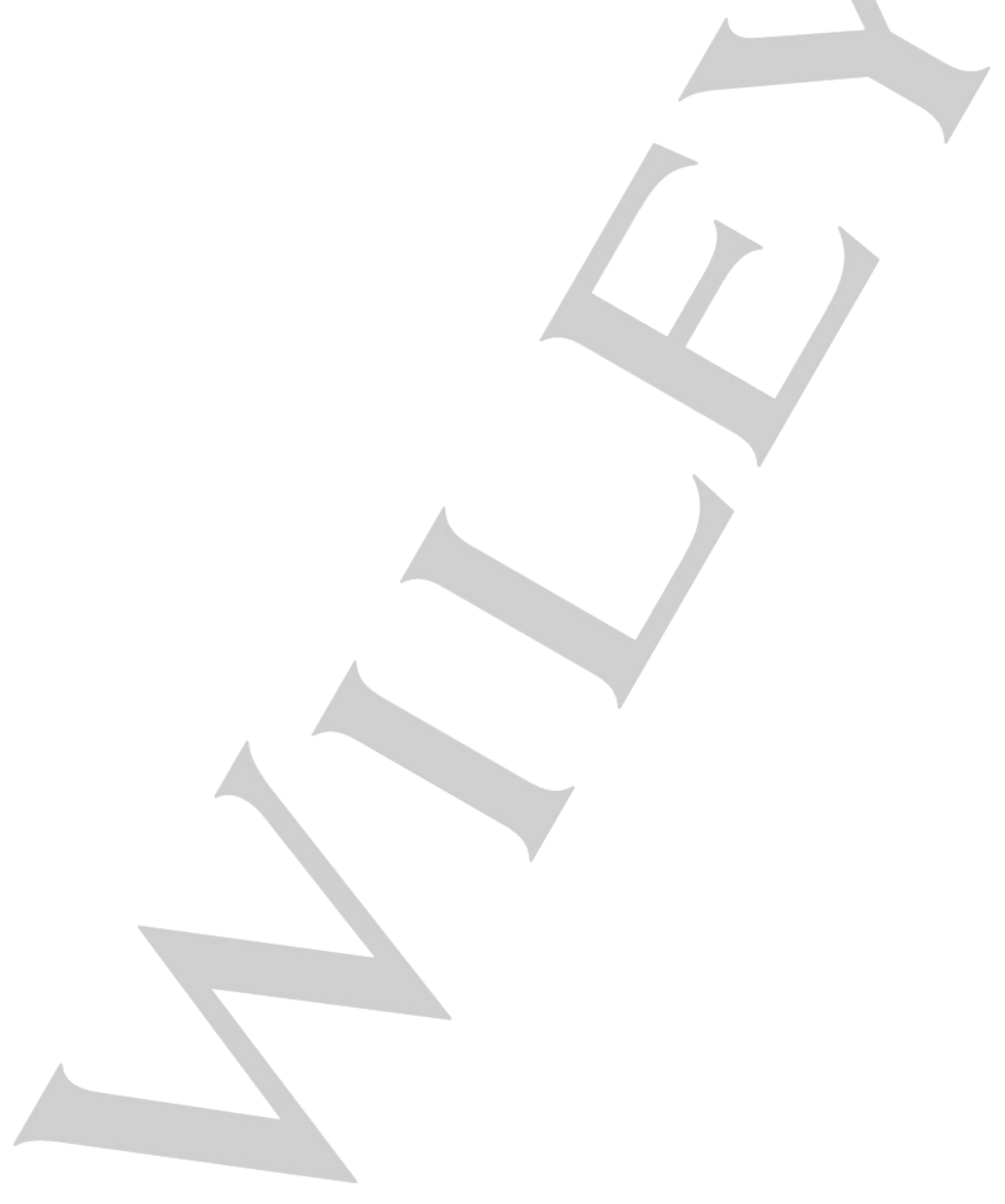

For internal use, please do not delete. Submitted_Manuscript

[a] Dr Clare F. Megarity, Dr Mary Clare Caraher, Dr David J. School of Biological Sciences, Queen's University Belfast, Biology Centre, 97 Lisburn Road, Belfast, BT9 7BL, UK

[b] Dr Hoda Abdel-Aal Bettley, Dr M. Clare Caraher, Dr Kathe Scott, Dr Richard A. Bryce, Dr Karen A. Nolan, Prof Ian J. Manchester Pharmacy School, [c] Dr Roger A. Whitehead, of Chemistry, and [d] DrThomas A. Jowitt, The Faculty of $L$ Science, Manchester Cancer Research Centre and the Un 